\title{
Interaktion von Patient und Ventilator während nichtinvasiver druckunterstützter Spontanatmung bei Patienten mit hyperkapnischer COPD ${ }^{1}$
}

\begin{abstract}
Zusammenfassung: Hintergrund: Die nicht-invasive druckunterstützte Spontanatmung („pressure support ventilation“, NPSV) erfordert das aktive Auslösen der Beatmung bei jedem Atemzug. Die Studie sollte ermitteln, wie sich NPSV, auf das Atemmuster, die Ventilation und die Belastung der Atemmuskulatur bei Patienten mit stabiler hyperkapnischer chronischobstruktiver Lungenerkrankung (COPD) auswirkt. Methode: 7 Patienten (Alter $66 \pm 9$ Jahre; $\mathrm{FEV}_{1} 43 \pm 13 \%$ Soll; $\mathrm{PaO}_{2}$ $52 \pm 19 \mathrm{mmHg} ; \mathrm{PaCO}_{2} 58 \pm 12 \mathrm{mmHg}$ ) nahmen an der Studie teil. Die physiologischen Variablen wurden bei Spontanatmung und am Ende einer 60-minütigen NPSV-Beatmung ermittelt. Die Beatmung erfolge über eine Gesichtsmaske. Der Inspirationsdruck (IPAP) wurde auf $12-14 \mathrm{~cm} \mathrm{H}_{2} \mathrm{O}$, der Exspirationsdruck (EPAP) auf $3 \mathrm{~cm} \mathrm{H}_{2} \mathrm{O}$ eingestellt. Die Aktivität der Atemmuskeln wurde als ösophageales und transdiaphragmales Druck-Zeit-Produkt (PTPes und PTPdi) gemessen. Ergebnisse: Ineffiziente Atemmuskeltätigkeit (Non-triggering) wurde nur sporadisch beobachtet. NPSV verbesserte die Ventilation im Vergleich zur nicht-unterstützten Spontanatmung: der $\mathrm{PaCO}_{2}$ sank von $58 \pm 11 \mathrm{mmHg}$ auf $50 \pm 14 \mathrm{mmHg}(p<0,05)$. Gleichzeitig wurde die Atemmuskulatur um $30 \%$ entlastet $(p<0,05)$. Die Atemfrequenz und das Atemmuster änderten sich nicht. Schlussfolgerungen: Bei klinisch stabilen Patienten mit hyperkapnischer COPD wird durch NPSV die Atemanstrengung zuverlässig erkannt und unterstützt. Dadurch verbessert sich die Ventilation und die Atemmuskulatur wird entlastet.
\end{abstract}

Patient-ventilator Interaction with Noninvasive Pressure Support Ventilation in Patients with Hypercapnic COPD Background: Noninvasive pressure support ventilation (NPSV) demands triggering with each breath. This study investigates the effects of NPSV via face mask on breathing pattern, ventilation and respiratory muscle loading in patients with hypercapnic stable COPD. Methods: 7 patients (age $66 \pm 9$ years; $\mathrm{FEV}_{1}$ $43 \pm 13 \%$ predicted; $\mathrm{PaO}_{2} 52 \pm 19 \mathrm{mmHg} ; \mathrm{PaCO}_{2} 58 \pm 12 \mathrm{mmHg}$ ) were included. The physiologic variables were evaluated during spontaneous breathing and at the end of a 60 minutes period with NPSV. Inspiratory positive airway pressure (IPAP) and expiratory positive airway pressure (EPAP) were adjusted to 12-

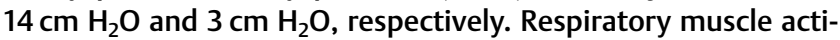
vity was measured as esophageal and transdiaphrgmatic pressure time product (PTPes and PTPdi). Results: Non-triggering was observed only occasionaly. Compared to unsupported

Pneumologie 2001; 55: 7-12

(c) Georg Thieme Verlag Stuttgart · New York ISSN 0934-8387
W. Pankow, H. Becker, U. Köhler, H. Schneider, T. Penzel, J. H. Peter

Zentrum für Innere Medizin, Abteilung Medizinische Poliklinik, Zeitreihenlabor, Philipps-Universität, Marburg

spontaneous breathing NPSV improved ventilation: $\mathrm{PaCO}_{2}$ was reduced from $58 \pm 11 \mathrm{mmHg}$ to $50 \pm 14 \mathrm{mmHg}(p \pm 0,05)$. Respiratory muscles were unloaded by $30 \%(p \pm 0,05)$. Breathing frenquency and breathing pattern did not change. Conclusions: In patients with hypercapnic stable COPD NPSV effectively recognizes and supports breathing efforts. As a result ventilation is improved and respiratory muscles are unloaded.

\section{Einleitung}

Bei Patienten mit schwergradiger COPD ist die Sauerstofflangzeitherapie bisher die einzige Therapieform, die einen gesicherten Einfluss auf die Prognose hat [1,2]. Der Stellenwert der nicht-invasiven Heimbeatmung ist dem gegenüber noch unklar [3]. Während bei hyperkapnischen Patienten mit COPD in einer Studie kein Vorteil gegenüber der Sauerstofflangzeittherapie im Langzeitverlauf gefunden wurde [4], erwies sich in einer anderen Untersuchung die nicht-invasive Beatmung plus Sauerstoff einer alleinigen Sauerstofftherapie als überlegen [5].

Vor dem Hintergrund der folgenden pathophysiologischen Überlegungen könnte sich die Heimbeatmung als günstig erweisen. Die alveoläre Hypoventilation bei Patienten mit fortgeschrittener COPD kann als Entlastungsstrategie für die durch die hohe mechanische Impedanz des respiratorischen Systems belastete Atemmuskulatur betrachtet werden [6]. Normoventilation würde die Atemmuskulatur überfordern. Daher wurde auch vorgeschlagen, den Begriff des „wise fighters“ [7] zu benutzen. Allerdings wird die Entlastung der Atemmuskeln mit negativen physiologischen und klinischen Folgen erkauft, die in erster Linie durch die Hypoxämie bedingt sind. Maschinelle Beatmung hat das Potenzial, die Atemmuskulatur zu entlasten und die Blutgase zu verbessern [8].

Bei NPSV muss im Gegensatz zur kontrollierten Beatmung jede Aktion des Ventilators aktiv ausgelöst werden (Triggern) und erfordert daher ein gewisses Maß an Aktivität der Atemmuskulatur. Die Entlastung der Atemmuskeln hat zur Voraussetzung, dass im Anschluss an den Trigger-Vorgang der Atemantrieb abgeschaltet wird. Inwieweit dies tatsächlich

${ }^{1}$ Herrn Prof. Dr. Peter von Wichert zum 65. Geburtstag gewidmet 
geschieht, hängt von den Details des Beatmungsmodus ab. Allerdings ist dieser Effekt schwer vorhersagbar. Deshalb untersuchten wir in der vorliegenden Studie den Einfluss von NPSV auf die patientenseitige Atemarbeit und auf die Blutgase. NPSV wurde dabei in Form von biphasischem CPAP (bilevel CPAP) appliziert. Dieses Beatmungsverfahren wurde gewählt, weil es bei der Heimbeatmung häufig eingesetzt wird und weil es durch einen empfindlichen Flow-Trigger das Potenzial hat, die „Triggerarbeit“ zu minimieren.

\section{Material und Methoden}

\section{Patienten}

Untersucht wurden Patienten, die wegen Exazerbation einer COPD mit respiratorischer Globalinsuffizienz in stationärer Behandlung waren. Die anthropometrischen Daten und die Ursachen der Exazerbation sind in Tab.1 aufgeführt. Zum Zeitpunkt der Untersuchung waren die Patienten in einem klinisch stabilen Zustand, so dass keine invasive Beatmung indiziert war. Ausdruck dafür war neben der Klinik der kompensierte Säure-Basen-Status. Alle Patienten gaben vor der Untersuchung ihr schriftliches Einverständnis. Das Untersuchungsprotokoll war zuvor von der Ethikkommission der Philipps-Universität Marburg akzeptiert worden.

\section{Beatmung}

NPSV wurde mit einem Vorserienmodell der Firma Respironics, Murrysville, PA, USA, durchgeführt. Dabei handelt es sich um einen miniaturisierten Ventilator mit der Option für mehrere Beatmungsmodi. Der hier verwandte Modus entsprach dem des in der Heimbeatmung häufig eingesetzten BiPAP-S der gleichen Firma. Die Druckunterstützung erfolgt dabei über das patientengetriggerte Umschalten zweier Druckniveaus. Das Gerät hat ein sehr empfindliches atemflussgesteuertes Ventil, das zwischen In- und Exspiration unterscheidet. Allerdings sind die Flusscharakteristika so modifiziert, dass das beim BiPAP-S häufig beobachte AutoTriggering nicht auftritt. Die Beatmung erfolgte über eine Nasen-Mund-Maske (Respironics, Murrysville, PA, USA). Die inspiratorische Druckunterstützung (IPAP) wurde auf 12$14 \mathrm{~cm} \mathrm{H}_{2} \mathrm{O}$ eingestellt. Dieser Inspirationsdruck wird nach eigener Erfahrung von den meisten Patienten gut vertragen.
Im Einzelfall wäre eine größere Druckunterstützung möglich gewesen. Es sollte jedoch bei allen Patienten der gleiche Druck verwandt werden. Das exspiratorische Druckniveau (EPAP) wurde auf $3 \mathrm{~cm} \mathrm{H}_{2} \mathrm{O}$ eingestellt.

\section{Messmethodik}

Die Messmethodik wurde in einer früheren Publikation detailliert beschrieben [9]. Daher erfolgt hier nur eine kurze Darstellung. Der Atemfluss wurde mit einem an der Maske befestigten Pneumotachographen (Bicore, Irvine, CA, USA) bestimmt. Der Druck an der Atemwegsöffnung wurde an der Gesichtsmaske als Pmask gemessen. Die Aktivität der Atemmuskulatur wurde mit Hilfe einer Messsonde ermittelt, die durch eine an der Maske angebrachte Öffnung geschoben wurde. Diese Sonde war bestückt mit zwei piezoelektrischen Druckaufnehmern, positioniert an der Spitze zur Ermittlung des gastralen Drucks (Pga) und $20 \mathrm{~cm}$ proximal davon zur Ermittlung des ösophagealen Drucks (Pes) (GaelTec, Dunvegan, Isle of Skye, GB). Die Messsonde eignet sich gut für atemmechanische Untersuchungen [10]. Die Sonde wurde über die Nase und den Ösophagus so weit vorgeschoben, bis beide Drucksensoren im Magen positioniert waren und anschließend so weit zurückgezogen, bis beim Einatemmanöver gegensätzliche Ausschläge der beiden Drucksignale als Hinweis auf die Lage des Pes-Sensors am gastroösophagealen Übergang registriert wurden. In liegender Körperposition mit leicht angehobenem Oberkörper wurde der Katheter weitere 10 bis $15 \mathrm{~cm}$ zurückgezogen, bis Artefakte durch Herzaktionen auf dem Pes-Signal minimal und Korrelationen zwischen den Schwingungen von Pes und Pmask beim Okklusionstest [11] optimal waren. Der Okklusionstest diente auch als Probe für die Maskendichtigkeit. Die Atemflusssignale und die Drucksignale wurden mit einer Frequenz von $100 \mathrm{~Hz}$ abgetastet und nach Transformation mit einem 12-bit Analog-Digitalkonverter mit einem von der Marburger Arbeitsgruppe entwickelten Computerprogramm (Topas) aufgezeichnet.

\section{Protokoll}

Die Untersuchungen erfolgen im Wachzustand in Rückenlage mit leicht angehobenem Oberkörper. Während einer 15minütigen Dauer wurde zunächst die spontane Atmung mit Maske ohne Anschluss an das Beatmungsgerät und ohne

Tab. 1 Anthropometrische und klinische Daten des untersuchten Kollektivs. BMI: Bodymass-Index; AF: Atemfrequenz, FEV ${ }_{1}$ : 1-SekundenKapazität; FVC: Forciertes Expirationsvolumen; n. u. nicht untersucht

\begin{tabular}{|c|c|c|c|c|c|c|c|c|}
\hline $\begin{array}{l}\text { Patient/Geschlecht/ } \\
\text { Alter (Jahre) }\end{array}$ & $\begin{array}{l}\mathrm{BMI} \\
\left(\mathrm{kg} / \mathrm{m}^{2}\right.\end{array}$ & Ursache der Exazerbation & $\begin{array}{l}\text { AF } \\
(1 / \min )\end{array}$ & $\mathrm{pH}$ & $\begin{array}{l}\mathrm{PaO}_{2} \\
(\mathrm{mmHg})\end{array}$ & $\begin{array}{l}\mathrm{PaCO}_{2} \\
(\mathrm{mmHg})\end{array}$ & $\begin{array}{l}\text { FEV }_{1} \\
\% \text { Soll }\end{array}$ & $\begin{array}{l}\mathrm{FEV}_{1} / \mathrm{FVC} \\
\%\end{array}$ \\
\hline $1 / \mathrm{M} / 71$ & 26,1 & akute Bronchitis, Linksherzinsuffizienz & 24 & 7,47 & 54 & 45 & 32 & 40 \\
\hline $2 / \mathrm{M} / 67$ & 30,5 & akute Bronchitis & 20 & 7,38 & 60 & 53 & 36 & 41 \\
\hline $3 / F / 73$ & 25,8 & Linksherzinsuffizienz & 23 & 7,39 & 65 & 76 & 36 & 51 \\
\hline $4 / F / 51$ & 40,3 & akute Bronchitis, Linksherzinsuffizienz & 34 & 7,36 & 26 & 70 & 33 & 59 \\
\hline $5 / \mathrm{M} / 73$ & 35,5 & akute Bronchitis, Lungenembolie & 28 & 7,42 & 42 & 62 & 56 & 62 \\
\hline $6 / \mathrm{M} / 70$ & 34,3 & Linksherzinsuffizienz & 16 & 7,39 & 38 & 51 & 62 & 68 \\
\hline $7 / \mathrm{M} / 57$ & 37,2 & akute Bronchitis & 16 & 7,39 & 81 & 50 & n.u. & n.u. \\
\hline Mittelwert 66,0 & 32,8 & & 23,0 & 7,400 & 52,3 & 58,1 & 42,5 & 53,5 \\
\hline Standardabweichung 8,6 & 5,5 & & 6,0 & 0,033 & 18,5 & 11,5 & 13,0 & 11,5 \\
\hline
\end{tabular}




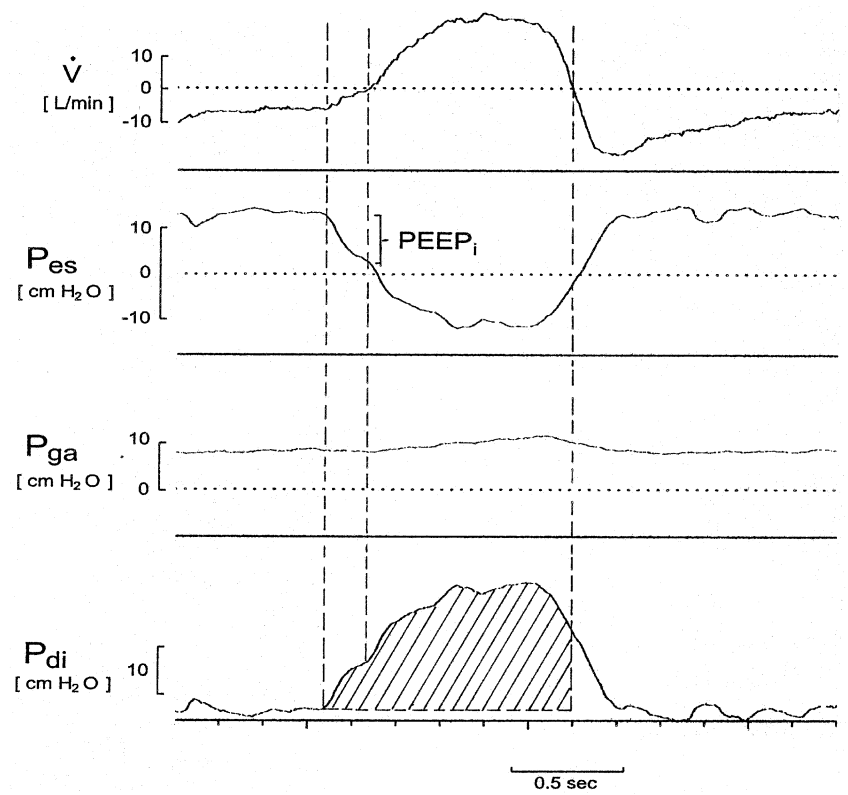

Abb. 1 Demonstriert wird die Berechnung des intrinsischen positiven end-exspiratorischen Drucks (PEEPi) und des inspiratorischen transdiaphragmalen Druck-Zeit-Produkts (PTPdi). Die erste gestrichelte vertikale Linie markiert den Beginn der Inspirationsanstrengung, erkennbar am steilen Abfall des Ösophagusdrucks (Pes) und am steilen Anstieg des transdiaphragmalen Drucks (Pdi). Der Beginn des inspiratorischen Atemflusses ( $\dot{V}$ ) ist zeitlich verzögert (zweite gestrichelte vertikale Linie). Der Druckabfall von Pes zwischen diesen beiden Linien entspricht PEEPi. PTPdi ist die Fläche unter der Pdi-Kurve vom Beginn der inspiratorischen Aktivität des Zwerchfells (erste gestrichelte vertikale Linie) bis zum Beginn der Ausatmung (dritte gestrichelte vertikale Linie).

Verbindungsschläuche aufgezeichnet. Anschließend erfolgte eine 60-minütige Beatmungsperiode mit NPSV. Blutproben für die Blutgasanalyse wurden vor und während der letzten Minuten unter Beatmung aus dem hyperämisierten Ohrläppchen entnommen.

\section{Berechnungen}

Leckagen an der Gesichtsmaske machten eine genaue Registrierung des Atemflusses nur bei Spontanatmung möglich. Daher konnte das Atemzugvolumen (VT) während der Beatmung nicht bestimmt werden. Inspirationszeit (TI) und
Exspirationszeit (TE) wurden anhand des Atemflusssignals ermittelt. Die Berechnungen des dynamischen intrinsischen positiven end-exspiratorischen Drucks (PEEPi) und der Atemmuskeltätigkeit ist in Abb.1 erklärt. PEEPi wurde als Druckdifferenz des Ösophagusdrucks vom Beginn des steilen Druckabfalls von Pes bis zum Beginn des inspiratorischen Atemflusses gemessen. Als Maß der Atemmuskeltätigkeit dienten die inspiratorischen Druck-Zeit-Integrale des Ösophagusdrucks (PTPes) und des transdiaphragmalen Drucks (PTPdi). Dabei wurde elektronisch die Fläche über der Pes-Kurve bzw. unter der Pdi-Kurve ermittelt: vom Beginn des steilen Abfalls der Pes-Kurve, der den Beginn der inspiratorische Muskelaktivität kennzeichnet, bis zum Ende der Inspiration beim Durchtrittspunkt des Atemflusssignals durch die Null-Linie. Der transdiaphragmale Druck (Pdi) berechnete sich dabei aus der Differenz von Pga und Pes. Die Druck-Zeit-Integrale wurden für die Dauer von 1 Minute als PTPes bzw. als PTPdi kalkuliert. Pes gilt hinsichtlich der Druckschwankungen als Maß für den Pleuradruck und beschreibt die Kraft, die durch die Summe der an der Atmung beteiligten Muskeln zur Dehnung der Lunge aufgebracht wird. Die zur Bewegung des Brustkorbs erzeugte Kraft wird dabei nicht erfasst. Pdi beschreibt spezifisch die Kraft, die durch den wichtigsten Atemmuskel, das Zwerchfell, erzeugt wird. Physiologische Untersuchungen haben gezeigt, dass PTPdi gut mit dem Sauerstoffverbrauch der Atemmuskulatur korreliert [12]. Die Daten aus mindestens 10 Atemzügen während Spontanatmung ohne Druckunterstützung und während der letzten 5 Minuten unter maschineller Beatmung wurden gemittelt. Die Berechnung der Unterschiede der physiologischen Variablen erfolgte mit dem Wilcoxon Rang-Summen-Test. Dabei wurde eine Irrtumswahrscheinlichkeit von weniger als $5 \%(p<0,05)$ als signifikant angesehen.

\section{Ergebnisse}

Von den 7 untersuchten Patienten waren 5 deutlich übergewichtig, 2 normalgewichtig. 2 der übergewichtigen Patienten hatten zusätzlich eine obstruktive Schlafapnoe. Alle Patienten tolerierten die Beatmung gut. Der IPAP betrug $12,5 \pm 1,0 \mathrm{~cm} \mathrm{H}_{2} \mathrm{O}$, EPAP $2,5 \pm 0,5 \mathrm{~cm} \mathrm{H}_{2} \mathrm{O}$. Die physiologischen Messergebnisse sind in Tab. 2 dargestellt. Die Atemfrequenz und das Atemmuster (Ti/Tot) änderten sich unter Beatmung nicht signifikant, obwohl in der Tendenz die Atemfrequenz etwas geringer war. Die Ventilation verbesserte sich, erkennbar am niedrigeren $\mathrm{PaCO}_{2}$. PTPes und PTPdi waren unter Beatmung etwa 30\% kleiner als bei Spontanatmung. Somit

\begin{tabular}{llll}
\hline & Spontanatmung & NPSV & Signifikanz \\
\hline $\mathrm{AF}(1 / \mathrm{min})$ & $24,4 \pm 5,8$ & $22,4 \pm 5,3$ & n.s. \\
$\mathrm{Ti} / \mathrm{Tot}$ & $0,38 \pm 0,05$ & $0,41 \pm 0,05$ & n.s. \\
PEEPi $\left(\mathrm{cm} \mathrm{H}_{2} \mathrm{O}\right)$ & $8,8 \pm 0,29$ & $7,6 \pm 3,2$ & n.s. \\
PTPes $\left(\mathrm{cm} \mathrm{H}_{2} \mathrm{O}^{*} \mathrm{~s}\right)$ & $418 \pm 86$ & $278 \pm 105$ & $\mathrm{P}<0,05$ \\
$\left.\mathrm{PTPdi}_{(\mathrm{cm} \mathrm{H}} \mathrm{O}^{*} \mathrm{~s}\right)$ & $445 \pm 89$ & $312 \pm 93$ & $\mathrm{P}<0,05$ \\
$\mathrm{PaO}_{2}(\mathrm{mmHg})$ & $57 \pm 16$ & $67 \pm 22$ & n.s. \\
$\mathrm{PaCO}_{2}(\mathrm{mmHg})$ & $58 \pm 11$ & $50 \pm 14$ & P $<0,05$ \\
pH & $7,40 \pm 0,05$ & $7,45 \pm 0,05$ & n.s. \\
\hline
\end{tabular}

Tab. 2 Einfluss von NPSV auf die Ventilation und die Aktivität der Atemmuskeln. AF: Atemfrequenz; Ti/Tot: Verhältnis Inspirationszeit/Atemzyklusdauer; PEEPi: intrinsischer positiver end-expiratorischer Atemwegsdruck; PTPes: ösophageales Druck-Zeit-Produkt; PTPdi: transdiaphragmales Druck-ZeitProdukt; n. s.: Unterschiede nicht signifikant. Weitere Erläuterungen siehe Text 

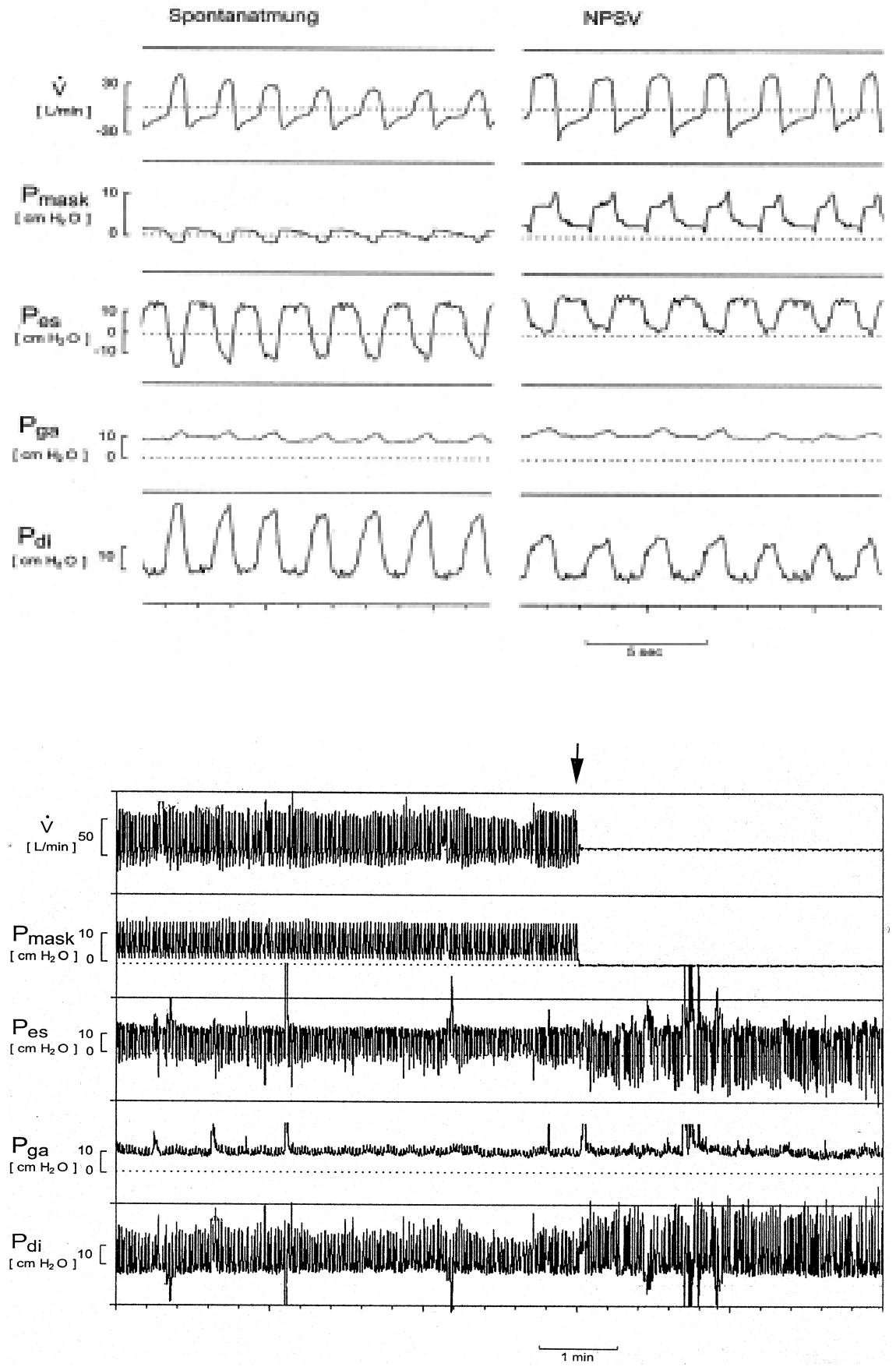

Abb. 2 Anhand eines repräsentativen Beispiels wird der entlastende Effekt der Beatmung auf die Atemmuskulatur demonstriert. Von oben nach unten sind folgende Parameter dargestellt: $\dot{V}$ : Atemfluss; Pmask: Maskendruck; Pes: Ösophagusdruck; Pga: gastraler Druck; Pdi: transdiaphragmaler Druck. Auf der linken Bildhälfte atmet Pat. 1 spontan durch die Maske ohne angeschlossenes Beatmungsgerät. Auf der rechten Bildhälfte ist die Spontanatmung durch NPSV unterstüzt. Dies ist erkennbar am Pmask. NPSV bewirkt eine deutliche Reduktion der Atemarbeit: die Amplituden von Pes und Pdi sind kleiner bei gleichzeitig verstärktem Atemfluss.

Abb. 3 Dargestellt wird beim gleichen Patienten wie in Abb. $\mathbf{2}$ der Übergang von NPSV (linke Bildhälfte) zur Spontanatmung ohne Druckunterstützung (rechte Bildhälfte). Der Pfeil markiert den Zeitpunkt, an dem die Maske abgesetzt wird. Erkennbar ist, dass nach Beendigung der maschinellen Beatmung die Belastung der Atemmuskulatur wieder zunimmt: die Amplituden von Pes und Pdi werden größer. bewirkte NPSV eine Teilentlastung der Atemmuskulatur. Abb. 2 und 3 verdeutlichen dies an Messbeispielen. Die Amplituden von Pes und Pdi sind mit NPSV deutlich kleiner als bei Spontanatmung ohne Druckunterstützung. Fehlendes Ansprechen des Beatmungsgeräts (Non-Triggering) ist beispielhaft in Abb. 4 dargestellt. Bei dem mit Pfeil gekennzeichneten Atemzug induziert der negative Ösophagusdruck im Gegensatz zu den übrigen Atemanstrengungen keine Druckunterstützung. Non-Triggering wurde aber nur selten beobachtet und war daher keine wesentliche Belastung der Atemmuskulatur.

\section{Diskussion}

Die vorliegende Studie zeigt, dass NPSV bei Patienten mit hyperkapnischer COPD die Atemmuskulatur entlastet und die Ventilation verbessert. Dies bestätigt die Ergebnisse der Mehrzahl (13-18) aber nicht aller [19], früherer Studien. In der Regel wurden inspiratorische Hilfsdrücke zwischen 10 und $20 \mathrm{~cm} \mathrm{H}_{2} \mathrm{O}$ verwandt. NPSV bewirkte eine variable Reduktion der Atemarbeit zwischen 20 und 100\% der Ausgangswerte bei Spontanatmung. Dagegen konnten Lien et al. [19] die diaphragmale EMG-Aktivität während einer 40-minütigen Beatmung mit bilevel CPAP (IPAP $10 \mathrm{H}_{2} \mathrm{O}$, EPAP $2 \mathrm{~cm} \mathrm{H} \mathrm{H}_{2} \mathrm{O}$ ) nicht reduzieren. Die Entlastung der Atemmuskulatur um ca. $30 \%$ 


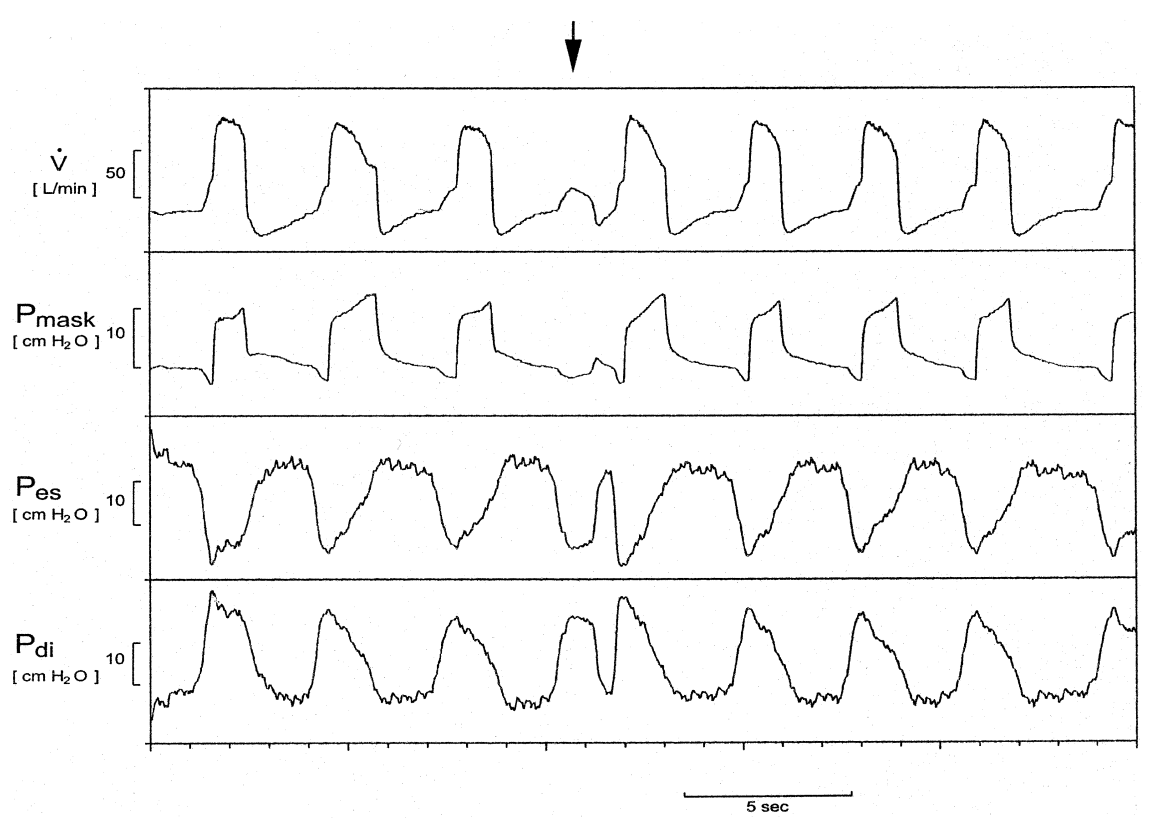

Abb. 4 Das Beispiel (Pat. 6) zeigt ein Ereignis (Pfeil), bei dem die inspiratorische Aktivierung der Atemmuskulatur keine Druckunterstützung durch den Ventilator auslöst (Non-Triggering). Die Atemanstrengung ist erkennbar an der Negativbewegung von Pes bzw. an der inversen Bewegung von Pdi. Im Gegensatz zu den benachbarten Atemzügen löst die Atemanstrengung keinen positiven Maskendruck (Pmask) aus. Derartige Ereignisse wurden aber nur selten beobachtet. war bei unserer Untersuchung geringer als bei einigen der zitierten Studien. Dies ist vermutlich durch drei Faktoren bedingt. Erstens waren die von uns verwandten Beatmungsdrücke relativ gering. Höhere Drücke hätten die Atemmuskulatur sicher stärker entlastet. Allerdings werden höhere Beatmungsdrücke von manchen Patienten nicht toleriert. Wegen der besseren Vergleichbarkeit wollten wir aber alle Patienten einheitlich beatmen und entschieden uns daher für relativ geringe Beatmungsdrücke.

Zweitens war die Wirkung der maschinellen Beatmung durch das Übergewicht der Mehrzahl der Patienten begrenzt. Die Beatmung muss dabei größere elastische Widerstände überwinden. Dazu sind größere Beatmungsdrücke notwendig als bei normalgewichtigen Patienten. 5 der 7 Patienten waren deutlich übergewichtig und zwei der Patienten hatten zusätzlich eine obstruktive Schlafapnoe. Die Kombination von COPD, Übergewicht und Schlafapnoe ist keineswegs ungewöhnlich. In einer großen Untersuchung an 311 Patienten mit COPD war unter den hyperkapnischen Patienten der Anteil Übergewichtiger deutlich erhöht [7]. In einer anderen Untersuchung wurden eukapnische und hyperkapnische COPD-Patienten mit vergleichbarer Atemwegsobstruktion verglichen. Die hyperkapnischen Patienten hatten ein deutlich größeres Körpergewicht. Außerdem waren in dieser Gruppe überwiegend starke Schnarcher mit vermehrten nächtlichen Hypopnoen und im Computertomogramm engeren oberen Atemwegen [20]. Übergewicht prädisponiert zur alveolären Hypoventilation vermutlich durch die additiven Effekte von elastischen und resistiven Widerständen auf Atemmuskulatur.

Drittens wird der Effekt der Beatmung auf die Atemarbeit durch den hohen intrinsischen PEEP begrenzt. PEEPi ist Bestandteil der erhöhten atemmechanischen Impedanz bei ausgeprägter COPD. Die großen Atemwegswiderstände und die geringere Elastizität des Lungengewebes bewirken, dass in der Exspirationszeit nicht vollständig bis zum funktionellen Residualvolumen ausgeatmet wird. Am Ende der Exspiration verbleibt ein positiver Retraktionsdruck des respiratorischen
Systems, der bei der folgenden Inspiration überwunden werden muss. Dieser Druck wird als PEEPi bezeichnet [21]. Bei der von uns untersuchten Gruppe war PEEPi deutlich größer als bei früheren Untersuchungen [7,17]. Dies hängt wahrscheinlich mit dem Übergewicht in unserem Kollektiv zusammen. In einer früheren Studie konnten wir nachweisen, dass auch Adipositas, besonders in liegender Körperlage, den exspiratorischen Atemfluss limitieren und intrinsischen PEEP induzieren kann [22]. Demnach ist der hohe PEEPi in unserem Patientenkollektiv vermutlich durch die additiven Wirkungen von COPD und Adipositas auf die Atemmechanik bedingt. PEEPi wirkt atemphysiologisch als elastischer Schwellenwiderstand, der überwunden werden muss, bevor ein negatives Druckgefälle zwischen Alveolarraum und Atemwegsöffnung aufgebaut und damit inspiratorischer Atemfluss erzeugt wird. Aus diesem Grund ist auch das „Triggern“ des Ventilators erschwert. PEEPi kann durch erhöhten expiratorischen Druck (extrinsischer PEEP oder EPAP) teilweise konterbalanziert werden [21]. Bei Beginn unserer Studie hatten wir nicht erwartet, dass der PEEPi in unserem Untersuchungskollektiv so groß sein würde. Daher war im Protokoll ein relativ geringer EPAP von nur $3 \mathrm{~cm} \mathrm{H}_{2} \mathrm{O}$ vorgesehen. Demzufolge war PEEPi während NPSV auch nicht geringer als unter Spontanatmung. Es ist bereits nachgewiesen worden, dass man die Atemmuskulatur stärker entlasten kann, wenn man EPAP additiv zu NPSV appliziert [17]. Wegen der inhomogenen Verteilung der intrapulmonalen Drücke sollte EPAP dabei $80 \%$ von PEEPi nicht überschreiten. Sonst wird die Exspiration erschwert und die Lungen können überbläht werden [21].

Für die nicht-invasive assistierte Beatmung gibt es bisher keine systematischen Untersuchungen zu der Frage, inwieweit ineffektive Atemanstrengungen durch fehlendes Triggern der Maschine auftreten. Bei unserer Untersuchung wurde zwar Non-Triggering vereinzelt beobachtet. Jedoch waren diese Ereignisse quantitativ zu vernachlässigen. Daraus lässt sich schließen, dass unsere Patienten die durch den PEEPi bedingte Triggerschwelle problemlos überwinden konnten. Bei noch schwergradiger COPD als bei unseren Patienten 
könnten hier aber Probleme auftreten. Die Triggerschwelle kann durch EPAP etwas unterhalb von PEEPi reduziert werden.

Alle von uns untersuchten Patienten ließen sich problemlos beatmen. Es ist bekannt, dass adipöse Patienten mit dem klinischen Bild des „blue bloater“ die nicht-invasive Beatmung oft besser tolerieren als normal- oder untergewichtige Patienten mit COPD [23,24]. Die Gründe dafür sind unklar. Es wurde bereits darauf hingewiesen, dass der klinische Stellenwert der Heimbeatmung bei COPD noch nicht geklärt ist. Kontrollierte prospektive Studien fehlen. Eine nicht-kontrollierte Studie aus den 80er Jahren konnte aber zeigen, dass übergewichtige Patienten mit COPD unter Langzeit-Heimbeatmung eine bessere Prognose haben [25]. In der vorliegenden Untersuchung wurden nun zunächst positive physiologische Effekte im experimentellen Kurzzeitversuch demonstriert.

\section{Literatur}

${ }^{1}$ Nocturnal Oxygen Therapy Trial Group. Continuous or nocturnal oxygen therapy in hypoxemic chronic obstructive lung disease: a clinical trial. Ann Intern Med 1989; 93: 391 - 398

${ }^{2}$ Report of the Medical Research Council Working Party. Longterm domiciliary therapy in chronic hypoxic cor pulmonale complicating chronic bronchitis and emphysema. Lancet 1981; 1: $681-686$

${ }^{3}$ Sneerson JM. The changing role of mechanical ventilation in COPD. Eur Respir J 1996; 9: 393 - 398

${ }^{4}$ Lin CC. Comparison between nocturnal nasal positive pressure ventilation combined with oxygen therapy and oxygen monotherapy in patients with severe COPD. Am J Respir Crit Care Med 1996; 154: $353-358$

${ }^{5}$ Meecham Jones DJ, Paul EA, Jones PW, Wedzicha JA. Nasal pressure support ventilation plus oxygen compared with oxygen therapy alone in hypercapnic COPD. Am J Respir Crit Care Med 1995; 152: 538 - 544

${ }^{6}$ Schönhofer B, Köhler D. Ventilatorische Insuffizienz und hyperkapnische Kompensation infolge chronisch belasteter „Atempumpe“. Dtsch Med Wschr 1994; 119: 1209-1214

${ }^{7}$ Begin P, Grassino A. Inspiratory muscle dysfunction and chronic hypercapnia in chronic obstructive pulmonary disease. Am Rev Respir Dis 1991; 143: 905-912

${ }^{8}$ Turkington PM, Elliott MW. Rationale for the use of non-invasive ventilation in chronic ventilatory failure. Thorax 2000; 55: 417 423

${ }^{9}$ Pankow W, Hijjeh N, Penzel T, Becker HF, Peter JH, v Wichert P. Influence of noninvasive positive pressure ventilation on inspiratory muscle activity in obese subjects. Eur Respir J 1997; 10 : $2847-2852$

10 Panizza J, Finucane KE. Comparison of balloon and transducer catheters for estimating lung elasticity. J Appl Physiol 1992; 72: $231-235$

${ }^{11}$ Baydur A, Behrakis KP, Zin WA, Jaeger M, Milic-Emili J. A simple method for assessing the validity of esophageal balloon technique. Am Rev Respir Dis 1982; 126: 788 - 791

12 Field S, Scani S, Grassino A. Respiratory muscle oxygen consumption estimated by the diaphragm pressure time-index. J Appl Physiol 1984; 57: 44-51

${ }^{13}$ Ambrosino N, Nava S, Bertone P, Fracchia C, Rampulla C. Physiologic evaluation of pressure support ventilation by nasal mask in patients with stable COPD. Chest 1992; 101: 385-391
${ }^{14}$ Carrey Z, Gottfried SB, Levy RD. Ventilatory muscle support in respiratory failure with nasal positive pressure ventilation. Chest 1990; 97: $150-158$

${ }^{15}$ Elliott MW, Mulvey DA, Moxham J, Green M, Branthwaite MA. Inspiratory muscle effort during nasal intermittent positive pressure ventilation in patients with chronic obstructive airway disease. Anaesthesia 1993; 48: 8-13

${ }^{16}$ Elliott MW, Aquilina R, Green M, Moxham J, Simonds AK. A comparison of different modes of noninvasive ventilatory support: effects on ventilation and inspiratory muscle effort. Anaesthesia 1994; 49: 279-283

${ }^{17}$ Nava S, Ambrosino N, Rubini F, Fracchia C, Rampulla C, Torri G, Calderini E. Effect of nasal pressure support ventilation and external PEEP on diaphragmatic activity in patients with severe stable COPD. Chest 1993; 103: 143-150

${ }^{18}$ Renston JP, DiMarco AF, Supinski GS. Respiratory muscle rest using nasal BiPAP ventilation in patients with stable severe COPD. Chest 1994; 105: $1053-1060$

${ }^{19}$ Lien T-C, Wang J-H, Chang M-T, Kuo C-D. Comparison of BiPAP nasal ventilation and ventilation via iron lung in severe stable COPD. Chest 1993; 104: 460-466

${ }^{20}$ Chan CS, Bye PTP, Woolcock AJ, Sullivan CE. Eucapnia and hypercapnia in patients with chronic airflow limitation. Am Rev Respir Dis 1990; 141: $861-865$

${ }^{21}$ Rossi A, Polese G, Brandi G, Conti G. Intrinsic positive endexpiratory pressure (PEEPi). Intensive Care Med 1995; 21: 522 536

22 Pankow W, Podszus T, Gutheil T, Penzel T, Peter JH, v Wichert P. Expiratory flow limitation and intrinsic positive end-expiratory pressure in obesity. J Appl Physiol 1998; 85: 1236-1243

${ }^{23}$ Ambrosino N, Foglio K, Rubini F, Clini E, Nasa S, Vitacca M. Noninvasive mechanical ventilation in acute respiratory failure due to chronic obstructive pulmonary disease: correlates for success. Thorax 1995; 50: $755-757$

${ }^{24}$ Schneider H, Becker H, Fus E, Stamnitz A, Peter JH, von Wichert P. Nasale positive Druckbeatmung (BiPAP) bei Patienten mit ausgeprägter chronisch obstruktiver Lungenerkrankung. Med Klin 1994; 89 (Suppl. 1): $71-73$

${ }^{25}$ Muir JF. Intermittent positive pressure ventilation (IPPV) in patients with chronic obstructive pulmonary disease (COPD). Eur Respir Rev 1992; 210: 335-345

\section{PD Dr. W. Pankow}

Krankenhaus Neukölln

Innere Medizin III

Rudower Straße 48

12351 Berlin

E-mail: pankow-berlin@t-online.de 